Stefan Biffl · Arndt Lüder Detlef Gerhard Editors

Multi-Disciplinary Engineering for
Cyber-Physical Production Systems

Data Models and Software Solutions for Handling Complex Engineering Projects

Springer 


\title{
Chapter 8 \\ Engineering of Next Generation Cyber-Physical Automation System Architectures
}

\author{
Matthias Foehr, Jan Vollmar, Ambra Calà, Paulo Leitão, \\ Stamatis Karnouskos, and Armando Walter Colombo
}

\begin{abstract}
Cyber-Physical-Systems (CPS) enable flexible and reconfigurable realization of automation system architectures, utilizing distributed control architectures with non-hierarchical modules linked together through different communication systems. Several control system architectures have been developed and validated in the past years by research groups. However, there is still a lack of implementation in industry. The intention of this work is to provide a summary of current alternative control system architectures that could be applied in industrial automation domain as well as a review of their commonalities. The aim is to point out the differences between the traditional centralized and hierarchical architectures to discussed ones, which rely on decentralized decision-making and control. Challenges and impacts that industries and engineers face in the process of adopting decentralized control architectures are discussed, analysing the obstacles for industrial acceptance and the new necessary interdisciplinary engineering skills. Finally, an outlook of possible mitigation and migration actions required to implement the decentralized control architectures is addressed.
\end{abstract}

Keywords CPS in production - Future automation systems • Industrial systems engineering $\bullet$ Migration strategy $\bullet$ System architectures

\footnotetext{
M. Foehr $(\square) \cdot$ J. Vollmar • A. Calà

Siemens AG Corporate Technology, Erlangen, Germany

e-mail: matthias.foehr@siemens.com; jan.vollmar@siemens.com; ambra.cala.ext@siemens.com

P. Leitão

Polytechnic Institute of Bragança, Bragança, Portugal

e-mail: pleitao@ipb.pt

S. Karnouskos

SAP, Walldorf, Germany

e-mail: stamatis.karnouskos@sap.com
}

\author{
A.W. Colombo \\ University of Applied Sciences Emden/Leer, Emden, Germany \\ e-mail: awcolombo@technik-emden.de
}




\subsection{Introduction}

Production systems are complex systems composed of various, often engineering discipline specific, subsystems. One important subsystem to be considered is the automation system. Due to the close interaction between the automation system and other system components like actuators and sensors the whole system and its environment needs to be considered when dealing with the automation system architecture. From this perspective the purpose (e.g., product to be produced) and system goal (e.g., output capacity) are main influencing factors. But also the overall system architecture (e.g., structure of production system, layout, IT-Systems) and the functional and non-functional requirements (e.g., degree of automation) towards the automation system have an impact on the automation infrastructure. Last but not least the available technology and hardware must be taken into account.

For today's systems the environment, system goals and system architecture are considered stable over the whole life-cycle of the production system. Changes occur when product changes (e.g., new model of car, new chemical substance) or requirements change (e.g., new safety regulation) but they normally have no impact on the architecture as such, except from the software and run-time aspects of the automation system. If changes occur, the production is stopped and the system is changed and production is re-started after modification. These downtimes, even if planned, are resulting in a loss of production capacity and finally a loss of money. This is also reflected in the classical automation system architecture as discussed in Sect. 8.2.

The question that arises is: are the above mentioned influencing factors going to remain stable also in the future? To answer it, the German National Academy of Science and Engineering (ACATECH, 2011) investigated four future scenarios of Cyber-Physical Systems (CPS) application with a time horizon until the year 2025.

One of these scenarios "Cyber-Physical System for the factory of the future" describes the characteristics and challenges for production systems. Production systems shall be able to react virtually in real time to changes in the market and the supply chain using CPS, which cooperate with ultra-flexibility even beyond company boundaries. Therefore a future industrial system architecture is needed that will focus on key aspects as identified in Kagermann et al. (2013), specifically:

- Allow flexibility and reconfiguration (with no downtime)

- Enable high production system resilience (deal with uncertainties)

- Enable continuous, automatic production optimization

- React faster and more automated to evolving customer and production demands

- Support for highly individualized production and small batches/lot sizes (lot size 1)

The posed requirements are reflected in several key research questions (RQs). However, most notably this chapter pertains aspects that tackle or enable approaches targeting the following RQs (see Chap. 1): 
- Modelling the structure and behaviour of Cyber-Physical Production Systems (CPPS) (RQ M1)

- Information integration in and across value chains (RQ I1)

- Description of plug-and-play capabilities and interfaces for engineering and run time (RQ I3)

- Modelling of CPPS flexibility and self-adaptation capabilities (RQ C1)

- Linking discipline-specific engineering views for flexible and self- adaptable CPPS (RQ C2)

The contributions of this chapter, are strongly liked to the emerging domain of CPS, and especially in their utilization in production systems. The discussions pertaining this chapter focus on providing an overview of automation system needs and evolution, how these are migrated to an new information-driven interoperable and service-enabled infrastructure, and what key considerations as well as challenges lie ahead. The intention in this chapter is not to provide a new model-based approach but to understand why and how the already existing methods and tools that enable production system flexibility and self-adaptation of CPPS are not adequate or too poorly implemented in industrial practice.

Based on discussed key requirements new automation system architectures are emerging in different research approaches which will be described in more detail in Sect. 8.2. As the design of completely new production systems, also referred to as green-field, is of secondary importance since a high number of legacy systems already exists, adequate migration strategies are needed to transform and migrate from existing automation system architectures to future ones. This transformation is described in Sect. 8.3. Furthermore the way to engineer these future automation systems has to be re-thought. This must include new methods and tools for engineers to design, implement and support such systems. Also educational programs have to be up-dated to ensure availability of experts that are capable to deal with these new systems architecture and new engineering paradigms. In Sect. 8.4 a closer look is taken upon these aspects. Finally, Sect. 8.5 gives an outlook and presents the main conclusions.

\subsection{The Evolution of Automation System Architectures}

Today companies are facing new market challenges in the manufacturing industry. In response to new requirements, innovative forms of manufacturing are recently introduced accordingly to the German "Industry 4.0" paradigm (Kagermann et al., 2013). The need of new manufacturing approaches is influenced by several aspects, namely market competitiveness, technology innovation, and customer requirements.

The global competition requires shortened delivery time and time-to-market, smaller lot sizes and shorter product life-cycle. Meanwhile, rapid changes in process technology force the fast integration of new functions into existing systems that are subject to obsolescence. Furthermore, customer expectations include not only lower 
prices but also more variety, higher quality and faster delivery of the product. In order to dynamically react to continuous changes of the business environment, the view on production system control must evolve.

The traditional production control systems are not able to support industries in overcoming such issues (Delsing et al., 2012). Centralized and hierarchical control architectures are characterized by rigid and top-down communication flows that do not enable the easy integration of new modules and, therefore, cannot cope with sudden and rapid changes. Considering all these aspects, new challenges for industries arise (Karnouskos et al., 2014a), and the next efforts attempt to introduce in industry a new production approach characterized by flexibility to different processing tasks, adaptability to changing production environment, and reconfigurability to enable these changes, while maintaining the security, safety and stability provided by classical production systems.

\subsubsection{Classical Automation System Architectures}

Traditional automation control systems are generally structured hierarchically or centralized, due to the complexity of automation tasks and interactions between components. According to the ISA-95/IEC 62264 (ANSI/ISA, 2010) standard, the main automation tasks are split in different layers of a pyramidal structure as shown in Fig. 8.1. The ANSI/ISA (2010) standard defines a model for exchanges of information between systems in five abstraction levels: Level 0-Field, Level 1-Control (PLC), Level 2-Process Control (SCADA), Level 3-Manufacturing Execution (MES) and Level 4-Enterprise Management (ERP).

The applications located on the different levels typically consider different time frames that range from months, weeks and days for the higher levels to hours, minutes, seconds and milliseconds for the lower levels. The first three levels perform the control function to execute the technological production processes. The field level uses actuators and sensors to measure, determine and display the equipment
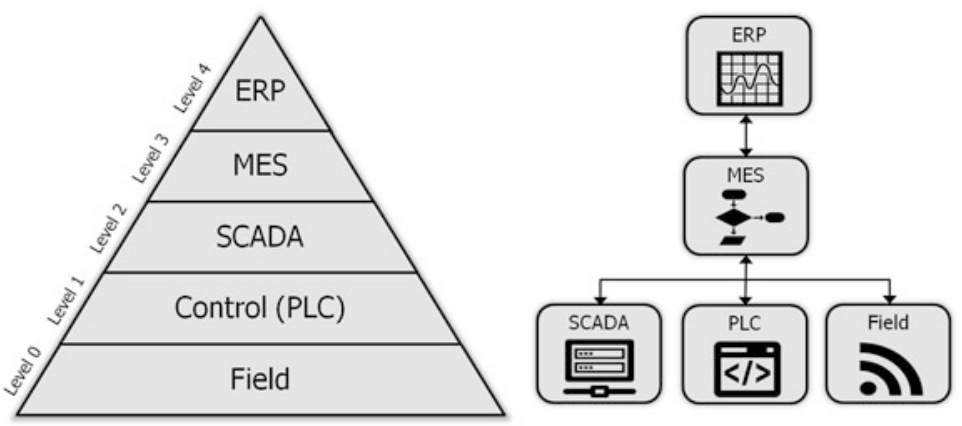

Fig. 8.1 ISA 95 hierarchical view of automation infrastructures 
data, while the control and process control levels are related respectively to the control of the product/process technology and to monitor the overall production system. Level 3 comprehends the activities of coordination and management of the production execution and, especially, the integration of different applications with respect to the main data and work flows. Level 4 is the highest level and represents the overall business management of the enterprise, including economical and logistic activities.

In system architectures structured according to ISA-95 the decision control is distributed among these hierarchical levels. This kind of structure has the advantages of predictability and robustness, as well good global optimization. It can be effective for small systems due to the characteristics of easy development and maintenance, and also adequate for systems running in very stable and structured environments. However, it is not adequate for emerging self-x automated manufacturing systems because of the insufficient adaptability and flexibility to production changes and the reduced performance in case of a single point of failure.

Analysing the scenario of "Cyber-Physical System for the factory of the future" (ACATECH, 2011), a "real-time" reaction of the production system to market changes cannot be performed by a hierarchical automation control system. In order to react more quickly to customer demands and environment changes, a more seamless integration of the automation pyramid's levels is required to change the production equipment and functions accordingly. The production units need to cooperate and organize themselves to optimize the production systems, saving time and costs. Capabilities, such as flexibility, adaptability and reconfigurability, are limited in a rigid communication structure with no cross-layer interoperability (Delsing et al., 2012), therefore, the traditional hierarchical ISA-95 structure needs to be transformed into a modular and flexible automation system architecture with decentralized control systems. The envisioned future production systems that possess self-x features, are cost efficient and easy to integrate at mass scale, cooperate in a cross-layer manner, interact with multiple stakeholders etc., justify the trend towards a distributed approach that is hardly or too costly to be realized with traditional approaches.

\subsubsection{Emerging Automation System Architectures}

The Cyber-Physical Systems (CPS) concept represents one of the key enablers of innovation in production systems accordingly with the Industry 4.0 paradigm. CPS focuses on the integration of logical and physical processes to control distributed physical systems, using cyber technologies (mechatronics, communication and information) (Lee, 2008; Leitão et al., 2016a). Since decades multi-agent systems (MAS) and service-oriented architecture (SOA) have been considered as the main approaches for implementing CPS and developing decentralized control systems in industry (Leitão and Karnouskos, 2015b). Several projects (Leitão et al., 2016b) have demonstrated their benefits. MAS is one of the most common approaches 
to realize decentralized control architectures by means of intelligent, modular and distributed agents that can be interconnected with physical hardware devices (Leitão et al., 2016a); and SOA is an architectural model for organizing and utilizing distributed capabilities in order to enable all components to communicate and interact via services (MacKenzie et al., 2006).

Next to these paradigms other concepts, such as plug-and-produce technology, web services and cloud manufacturing, have been investigated to build flexible and reconfigurable manufacturing control systems. During the last years a significant amount of research has been conducted and, recently, several European funded projects have reported important developments in this field and presented results at high technology readiness levels.

The GRACE-Integration of process and quality control using multi-agent technology_project (Castellini et al., 2011) developed, implemented and validated a cooperative MAS to integrate process control with quality control at local and global level. The MAS architecture was designed to manage the planned changes of set-point in production processes and the large variety of unforeseen disturbances and changes in process parameters and variables. Self-adaptation procedures and optimization mechanisms for process and product parameters were implemented and integrated into control and diagnostic systems at local level, in terms of individual agents, and global level, considering the data gathered in all the production system.

In parallel, the IDEAS-Instantly deployable evolvable assembly systemsproject (Onori et al., 2013) developed a fully distributed and pluggable environment capable to self-organize itself and control at the shop floor level using agent technology. The IDEAS assembly system ran with a multi-agent control setup and could be reconfigured on-the-fly assuring the integration of different self-configured modules at the shop floor in runtime. Moreover, the self-diagnosis capability of each module permits to have a distributed diagnosis and the entire system is capable of checking the propagation of problems and re-adapt whenever a component (module) is plugged without requiring programming effort in order to manage unpredicted behaviours.

Taking the experience from these projects, the PRIME-Plug and produce intelligent multi-agent environment based on standard technology-project (Antzoulatos et al., 2014) has gone one step forward to support assembly systems in distributed reconfiguration and monitoring. It developed a multi-agent architecture using plugand-produce principles for module integration, including legacy equipment, and methods for rapidly configuring production systems through innovative humanmachine interaction mechanisms. The PRIME approach is based on standard technologies (JADE multi-agent software framework, Vaadin and Cassandra database) and languages (JAVA and OPC-UA programs for interfacing and data exchange) for the integration and networking of heterogeneous control system from different equipment suppliers to support system evolution linked to process performance and product volume variability.

The I-RAMP ${ }^{3}$ - Intelligent reconfigurable machines for smart plug-and-produce production-project (Goncalves et al., 2014) focused on the transformation of 
conventional production equipment into network-enabled devices (NETDEVs). The NETDEV interface enables the integration of plug-and-produce devices and sensors and actuators at MES level for work flow optimization and production data assessment, using standardized communication and collaboration mechanisms.

The SOCRADES-Service-Oriented Cross-layer infRAstructure for Distributed smart Embedded Systems - project (Colombo and Karnouskos, 2009; Karnouskos et al., 2010; Colombo et al., 2010) used the Service-Oriented Architecture paradigm at device and application levels to build a design, execution and management platform for innovative industrial automation systems. The project focused on designing and implementing a cross-layer infrastructure that would enable the integration of industrial automation systems and devices up to the MES/ERP level (Karnouskos et al., 2007, 2009). The approach was driven by open standards, service-based integration, and collaboration among the various stakeholders, setting the stage for the next generation of automation systems (Colombo and Karnouskos, 2009).

The IMC-AESOP - Industrial Monitoring and Control ArchitecturE for ServiceOriented Process-project (Colombo et al., 2014b,a) used as a starting point the SOCRADES approach and extended it to realize cloud-based industrial CPS. Driven by key emerging information and communication technologies in industrial automation, and with a strong focus on the cloud (Karnouskos and Somlev, 2013), the project envisioned and realized an architecture (Karnouskos et al., 2014b) for industrial CPS automation infrastructures. The results have been demonstrated in the next generation cloud \& service based SCADA/DCS (Karnouskos and Colombo, 2011) for monitoring and control, including visions for their design, implementation, collaboration, and migration The architecture enables cross-layer service-oriented collaboration both at horizontal and vertical levels by utilizing service-oriented integration and the cloud.

The Self-Learning-Reliable Self-Learning production systems based on context-aware service - project (Stokic et al., 2011) proposed the service-oriented integration of different auxiliary processes into the main control. The processes are represented as services that fully interoperate in a Web Services platform. The Self-Learning system enables the reconfiguration of machines and processes based on user experiences acquired during the system runtime.

The FLEXA-Advanced flexible automation cell—project (Webb and Asif, 2011) developed a flexible manufacturing system based on web services architecture that connects the cell controller to ERP/MES.

The SelSus-Health Monitoring and Life-Long Capability Management for SelfSustaining Manufacturing Systems-project (Sayed et al., 2015) proposed a new paradigm for highly effective, self-healing production systems to maximize their performance over longer lifetimes using web-based services for multi-modal data acquisition techniques to validate, update and document all information on failure modes or degradation states.

The CassaMobile-Flexible Mini-Factory for local and customized production in a container-project (Friedrich et al., 2014) developed a new kind of local, flexible and environmentally friendly production system for highly customized 
Table 8.1 Overview of technologies in emerging automation system architectures

\begin{tabular}{|c|c|c|c|c|c|c|c|c|c|c|c|}
\hline $\begin{array}{r}\begin{array}{r}\text { Research } \\
\text { Projects }\end{array} \\
\text { Technologies }\end{array}$ & 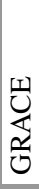 & 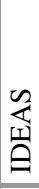 & 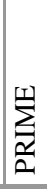 & 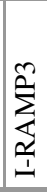 & 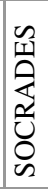 & 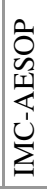 & 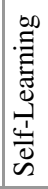 & $\begin{array}{l}\underset{x}{\mathbb{I}} \\
\text { 党 }\end{array}$ & $\begin{array}{l}\mathscr{n} \\
\frac{\mathscr{n}}{\mathscr{D}} \\
\tilde{n}\end{array}$ & 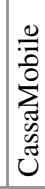 & 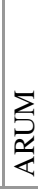 \\
\hline Multi-agent systems & - & • & $\bullet$ & & - & - & & & & & - \\
\hline SOA/Web services & & & & & $\bullet$ & - & $\bullet$ & • & () & • & $\bullet$ \\
\hline Cloud & & & & & (1) & - & & & $\bullet$ & & \\
\hline Plug-and-Play & & $\bullet$ & $\bullet$ & 0 & $\bullet$ & - & & & & $\bullet$ & \\
\hline Self-* features & $\bullet$ & $\bullet$ & 0 & (1) & ( & (1) & $\bullet$ & & $\bullet$ & $\bullet$ & \\
\hline
\end{tabular}

Legend: - covered; partially covered

parts based on a combination of different manufacturing processes. The production is based on a modular architecture that includes mechanical and control system adaptation by means of a SoA system.

One of the current trends in the future automation control research is to integrate these solution concepts in the same architecture. One example is the ARUMAdaptive Production Management—project (Leitão et al., 2013), which combined holonic multi-agent systems with services architecture using Enterprise Service Bus (ESB) to improve planning and control systems.

These projects show a transformation of the centralized architecture into a distributed control system using different technologies, as shown in Table 8.1. High levels of autonomy and cooperation of individual entities have been reached via multi-agent systems in which agents have their own intelligence and interact with each other optimizing their behaviour iteratively (Wooldridge, 2002; Leitão et al., 2016b). Service-oriented architecture technologies enable the integration of components that provide services to other components they are linked to, creating an Internet of Services for the production system. Web Services contain components description and exchange data information enhancing the vertical collaboration between device level and enterprise level. Moreover, hosting these services in a Cloud it is possible to rapidly compose new industrial application just by selecting and combining the information stored inside (Colombo et al., 2014b). Plug-and-play technologies are investigated to build modular structures that improve components interoperability and reusability to satisfy the requirement of rapid reconfigurability of the system (Antzoulatos et al., 2014). In addition, self-* capabilities support equipment integration, control and monitoring, as well as cooperation and adaptation.

Each of these projects provided an individual solution for flexible and reconfigurable distributed control architectures involving multi-agent systems (Leitão et al., 2016b), standard communication protocols, web services and Cyber-Physical components. However, these solutions solve only narrowed specific problems neglecting other technological issues. In order to facilitate a wider industrial uptake, the future industrial system architecture should be a result of the integration of these 


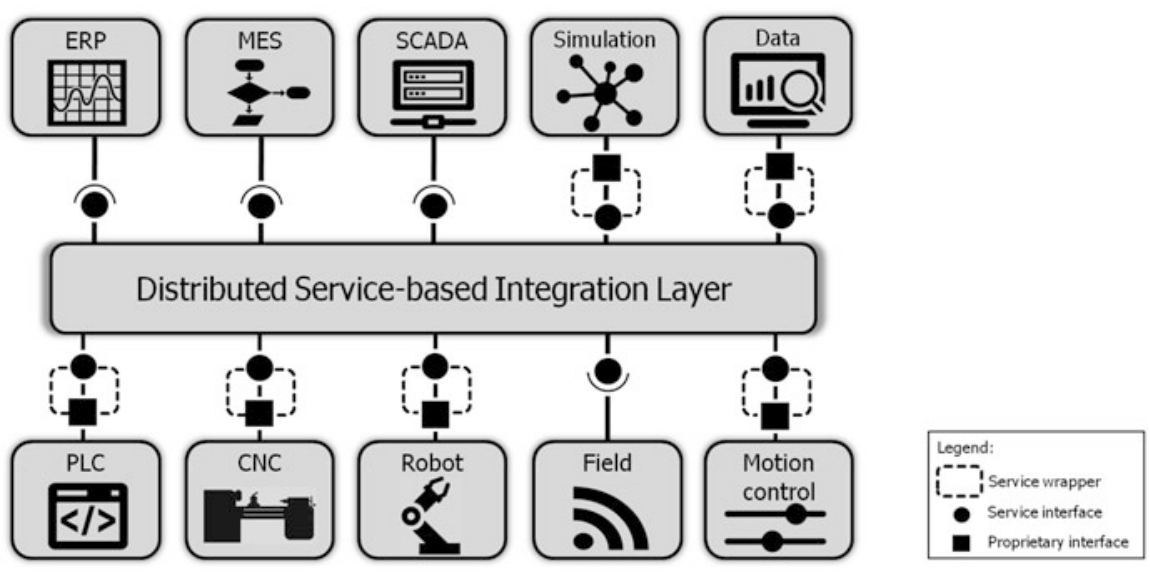

Fig. 8.2 Automation system integration vision over a common (service) infrastructure

technologies in a unique form (e.g., as shown in Fig. 8.2), covering the architecture, assets and process aspects of the overall production system. As an example, the SOCRADES project has demonstrated largely feasibility of this vision using web services for cross-layer integration and collaboration among devices, systems and other stakeholders (Colombo et al., 2010; Karnouskos et al., 2009; Taisch et al., 2009). A recent survey of acceptance factors of agent systems in industry (Leitão and Karnouskos, 2015a) sheds some additional light on key aspects that should be investigated at large when engineering of future industrial automation systems is to be considered.

In Fig. 8.2 a vision of automation system integration over a common servicebased infrastructure is proposed. A key role in this new vision is performed by the distributed service-based integration layer that aims to ensure the transparent, secure and reliable interconnection of the diverse heterogeneous hardware devices e.g., robotic cells and Programmable Logic Controllers (PLC), and software applications e.g., MES and SCADA/DCS (Karnouskos and Colombo, 2011). Current Business systems and higher-level applications (i.e., ERP and MES etc.) are typically fully service-based in their interactions with other systems. As such, integration with such systems is possible via services, and commonly via Internet technologies such as web services. However, any proprietary system, not providing service based interfaces, needs to be integrated via a service wrapper that translates proprietary interfaces in standard service based interfaces in order to connect the system to the other software applications and industrial hardware devices. An important innovation of this integration layer, e.g. developed in the PERFoRM project (PERFoRM, 2016a), is its distributed and cloud approach, instead of the centralized ones that can be mostly found nowadays and can act as a single point of failure as well as a limitation for the system scalability. For this purpose, this distributed integration layer handles the interconnection of these heterogeneous 
production components by following the service-orientation principles, i.e., each one is exposing their functionalities as services, which will be discovered and requested by the other components.

Since the implementation of new control technologies will have a direct impact on the production, the implementation of a new decentralized control architecture is not sufficient to achieve the exploitation of Cyber-Physical Production Systems (CPPS). A migration strategy that supports industries in adopting new technologies has been only partially considered in the past projects, e.g., IMC-AESOP envisioned the next generation SCADA/DCS systems (Karnouskos and Colombo, 2011) and investigated an approach to migrate SCADA and DCS systems to SOA (Delsing et al., 2011). At present, it is required a set of guidelines for engineers, equipment developers and end users to plan, support and realize an easy and smooth migration of the existing factories into the new generation of smart factories, taking into consideration both technical and economical issues.

\subsection{The Transformation of Automation System Architectures}

\subsubsection{Towards Information-Driven Automation Systems}

Business continuity and agility form the core modus operandi of modern global enterprises (Karnouskos, 2009), and efforts that yield results of more efficient automation systems are well-justified. In order to achieve the pursued agility and continuity, business processes performed in highly distributed production systems need to be efficiently integrated with a sophisticated shop-floor infrastructure that is capable of responding to dynamic adaptations in a timely manner (Karnouskos, 2011).

The prevalence of CPS and the advanced capabilities they offer, mean a drastic reshaping of the future automation system architectures. The increased complexity and sophistication of involved systems, make it very hard to follow monolithic and one-size-fits-all approaches, and make the transition towards modular, dynamic, and open systems imperative (Colombo and Karnouskos, 2009; Karnouskos, 2011). Over the last years, significant efforts have been realized towards service oriented architectures and systems that interact with them (Colombo et al., 2014b). The CPS principle pushes such limits even further, as CPS themselves as well as constellations of them and larger systems of systems need to adhere to similar design patterns and principles.

In such sophisticated infrastructures, emphasis is put on interaction of the CPS with its surrounding environment, which may dynamically change, and which is based on open technologies and interaction patterns rather than closed systems and proprietary software. Hence the integration aspects gain importance, and its focus is significantly expanded for large infrastructures and highly heterogeneous landscapes composed of thousands of devices, systems, services that need to 


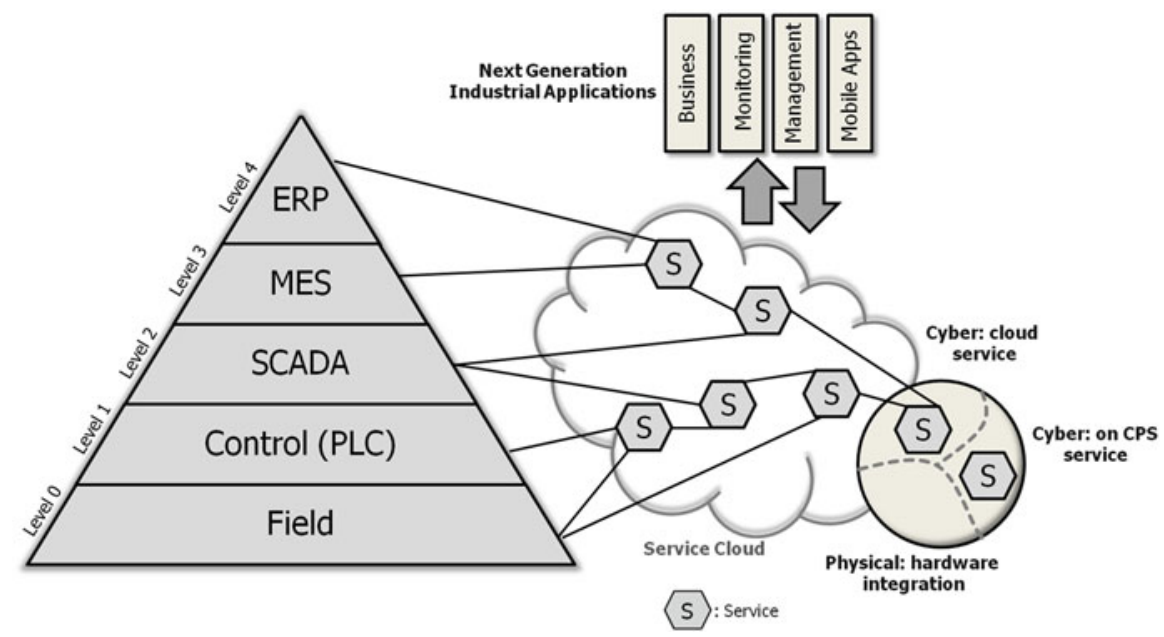

Fig. 8.3 Transitioning towards a SOA-based information-driven architecture by offering key functionalities as services (Karnouskos et al., 2014b; Colombo et al., 2014b)

interact, cooperate and realize their goals in an efficient manner (Colombo et al., 2013).

Considering envisioned architecture transitions such as the one shown in Fig. 8.3, the high-level changes imposed upon engineering of future automation systems are becoming easier to recognize. Figure 8.3 advocates that in parallel to traditional hierarchical architectures in industrial infrastructures, selected functionalities at different levels (e.g., as defined by the ISA-95 paradigm), can be exposed as a collection of CPS services. The latter, may exist in the CPS, traditional systems, as well as the cloud, giving rise to a highly heterogeneous, dynamic, and adequately performant ecosystem of services (Colombo et al., 2013; Karnouskos and Somlev, 2013). Upon such services, applications can cherry-pick the functionalities they need in order to rapidly and efficiently fulfil their goals.

It is important to notice that this transformation of automation systems is performed mainly at the virtual IT level and not in the physical counterpart of the system, which simplifies the migration from the existing automation production systems running currently in the factories to the future ones. Additionally, according to the McKinsey's report (McKinsey, 2015), the implementation of "industry 4.0" solutions will bring significant benefits with only about $40-50 \%$ of replacement of equipment.

Considering the proposed innovative automation systems architectures described in Sect. 8.2, one can identify some similarities among them. They build upon the distribution of control functions over intelligent, modular and cooperative entities providing modularity, flexibility, robustness, scalability and reconfigurability, which are at large weak aspects of traditional monolithic architectures. The distributed approach addresses the need to have adequate automation system architectures to 
tackle the scenario of "Factory of the Future", while being in-line with the guidelines defined by the "Industry 4.0" platform. These architectures also present intelligence and adaptation capabilities embedded in the distributed nodes and in the emergent system behaviour, and some exhibits evolution and self-* properties, such as selforganization, self-adaptation, self-optimization and self-healing.

The deployment of these new decentralized, smart automation architectures in industrial environments need to be performed in a smooth manner, transforming the solutions based on the traditional hierarchical ISA-95 automation structure into solutions based on a network of CPS (ACATECH, 2011; Leitão et al., 2016a). This transformation effort should consider the integration of heterogeneous robotics and automation machinery, as well as the existing legacy systems running in the current industrial solutions to avoid discontinuity and aiming a smooth migration. For this purpose, the plugability is simplified by considering proper industrial standards for protocols and technologies that enable easy integration and interaction among systems and services, while avoiding the creation of "technology islands".

\subsubsection{Migration Strategies}

The envisioned next generation of industrial automation architectures provide tangible benefits and are a good match for newly established infrastructures (greenfield projects) e.g., can be deployed in a new plant. However, the vast majority of existing infrastructures are brown field projects as they already have constraints in place (e.g., integration with legacy systems and processes), and need to go through migration stages, that will enable the smooth transition from existing systems to the sophisticated infrastructure envisioned.

Current lifetime of production facilities are long, and changes are infrequent and limited. However, this is increasingly changing and in conjunction with the prevalence of software and computational processing at the heart of the 4th industrial revolution, changes are going to be not sporadic but an integral part of the day-to-day business, transitioning towards a DevOps culture. As such, it can be considered that these changes will be applied through incremental migration steps, during the whole lifecycle of devices, services, systems and landscapes. This is especially important as plant operators typically invest multiple millions into their production systems. A change over to decentralized control by a complete revamp of the automation system in one big shot does not only yield a high risk of failure but also annihilates high amounts of investment before they repaid. A stepwise approach of system changeover can bring in small portions of the new distributed control at a time, reducing risks and also allowing to change over the system in accordance with investment ability of the plant operator. Hence, migration strategies are expected to play a pivotal role to the success of the envisioned infrastructures.

Considering the migration to an information-flat and service-based infrastructure as shown in Fig. 8.3, the steps that need to be undertaken are depicted at high level 


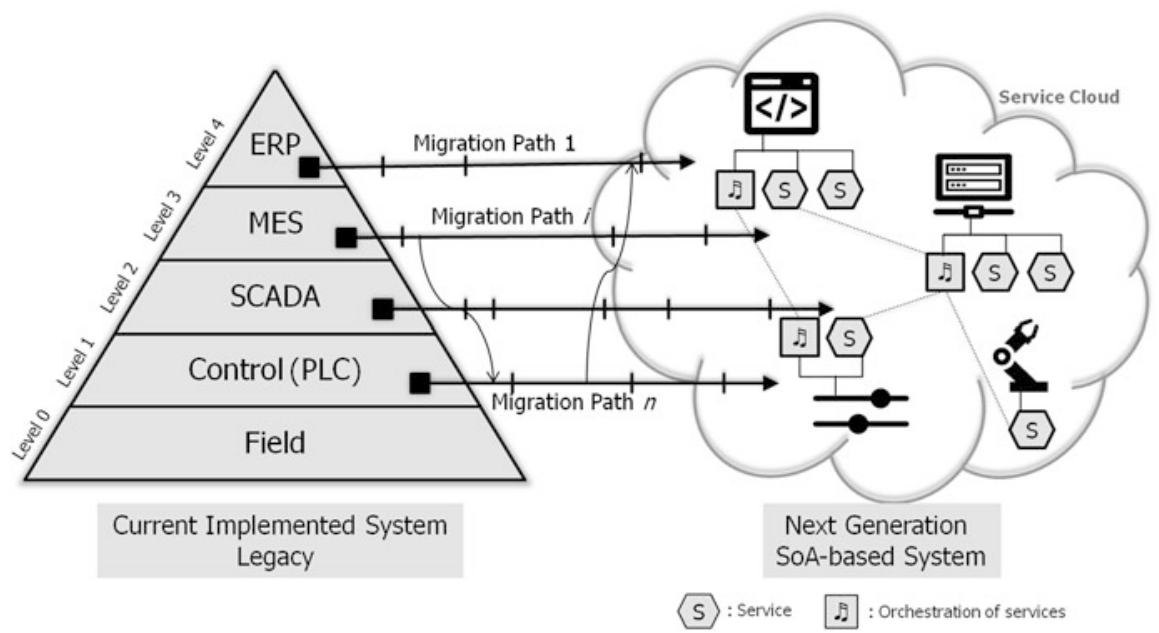

Fig. 8.4 Migration of complex functionalities and cross-layer dependencies to a full SOA-based Infrastructure (Colombo et al., 2014b; Delsing et al., 2011)

in Fig. 8.4. The different system characteristics prevalent in each ISA-95 layer, will need to be captured step-wise in services and be made available. However, as there are several inter-dependencies, the potential migration paths have to be assessed and a migration has to be done step-wise. In doing so, partially the new functionalities will become available to applications and services. Such migration will also unleash at system level emergent behaviours as a result of the dynamic interactions among the different devices and systems. Top-down and bottom-up approaches will need to be analysed in detail (Delsing et al., 2011), and the resulting migration strategies can be highly complex, depending on the preconditions, requirements and goals. More detailed examples with respect to migration and its challenges can be found in Colombo et al. (2014b). Figure 8.4 makes it clear that the migration is not an one-time operation, but rather a continuous one, that the automation industry will have to get accustomed to.

The software industry has long experience with step-wise development, release and upgrade of systems, and can manage such step-wise changes quite well. However, when it boils down to CPS infrastructures with strict operational and timing requirements, things are challenging. In addition, any migration strategies have a multitude of goals that go beyond technology and include, cost-effectiveness, resource-efficiency, agility, deterministic behaviour, operational easiness, business continuity etc. (Karnouskos, 2009). Due to their cross-disciplinary nature, applied at enterprise level, such migration strategies pose some risk which needs to be managed. However, once the envisioned architectures and modus operandi are in place, such incremental migratory actions are expected to be easier to realize. 


\subsection{Considerations on Future Automation System Architectures}

The transition from the existing traditional industrial automation systems, mainly based on Product-Life-Cycle (PLM) into the new CPS based approaches, should be smooth and requires a rethinking of engineering methodologies, integration of methods and tools from the different domains where the CPS are located and best practices. Since such changes have to consider also existing infrastructures and business continuity, consideration of migration and mitigation strategies to overcome the identified challenges is seen as of paramount importance. When talking about Engineering CPS-based Automation Systems, there are three main task clusters to consider:

- The Engineering to create new CPS components at device level (cyber- and physical views) and the Engineering to build the System of CPS.

- The Engineering to reconfigure or adapt an existing CPS, to operate it and to manage its evolution, both at device and system levels

- The Engineering to design, implement, operate and manage autonomous/smart CPS components within an intelligent automation infrastructure

\subsubsection{Rethinking of Automation Systems Engineering}

It is important to recognize that all the parts involved in future automation system architectures, will not be under the control of a single authority, and technology, and therefore, the integration, interaction and operation will need to be done via open interfaces exposed by the services (Karnouskos, 2011; Colombo et al., 2014b). Taking into consideration the goals of a CPS, as discussed in Sect.8.1, the engineering effort to adapt a CPS during run-time must be minimal. This means "Zero Engineering" during run-time must be prepared and implemented. The ability to reconfigure existing elements and to integrate new elements have to be a "builtin" capability of the CPS on system level. Engineering such systems has to cope with continuous updates of the infrastructure (both in hardware and software) and to provide high resilience for the CPS.

Aspects such as Systems of CPS integration and dynamic reconfiguration require a set of complementary engineering tasks, which are strongly related to the major characteristics to be covered by an adequate Systems-of-Systems engineering approach, i.e.,

- engineering evolvability at system level due to plug-and-play integration and live removal of CPS components;

- dynamic requirements engineering to support incremental live validation of structural and behavioural modifications of the system (understanding and managing "emergency"; 
- control re-configuration for several control systems that are strongly coupled;

- last but not least the integration of the human factor in each of the phases of the life cycle without loosing the System-of-Systems view perspective

As the different parts of such a system will evolve independently, good practices for engineering, upgrading, operating and maintaining them need to be followed.

The core idea behind the amalgamating the physical and virtual (digital) worlds, is to seamlessly gather useful data and information about objects of the physical world, transform it to knowledge, and empower various industrial applications (Karnouskos, 2011; Colombo et al., 2014b). The emerging engineering systems, operating in highly sophisticated infrastructures as discussed, are expected to enable the elimination of many existing pain points, but unavoidably it will create others. The new ones will require engineers to draw on knowledge from multiple disciplines (Broy and Schmidt, 2014; Karnouskos et al., 2014a) if they want to effectively capitalize on the new capabilities.

The automation engineers dealing with Industrial Systems of Cyber-Physical Systems have to possess a much wider set of skills to understand how the different constituent systems interact, both in structural and behavioural manner, as well as a solid background on Information, Communication, Control Technology and their fusion.

As such, engineering effective solutions implies e.g., technical excellence, understanding of hardware and software components in the infrastructure, knowledge of industrial operational context, understanding of interactions at device and system level, risk estimation, understanding of the impact of engineering decisions e.g., to safety, security, dependability, etc.

\subsubsection{Directions and Challenges}

The described transformation into the future industrial automation systems, and their industrial adoption, presents several challenges, which can be aggregated in 6 major clusters (Leitão et al., 2016a):

- CPS Capabilities, which comprises the modularization and servification of CPS systems, the development of CPS as System of Systems (SoS), their optimization and real-time monitoring and control, as well as the consideration of advanced (big) data analytics.

- CPS Management, which includes the security and trust in the management of large scale CPS, aiming to achieve industrially mature solutions.

- CPS Engineering, which comprises the safe programming and validation, the resilient risk mitigation, and methods and tools for the CPS and Systems-of-CPS life-cycle support, which are crucial challenges for the industry. A challenge is the need to apply new methods within the engineering of these systems (e.g., collaborative workflow generation and processing). 
- CPS Ecosystems, which includes the design and deployment of collaborative, autonomic, self-* and emergent CPS, as well as the integration of Humans in the Loop, many of them being expected to be matured only in the long-run.

- CPS Infrastructures, which are related to interoperability services, and mitigation and migration strategies to support the transformation of current automation systems into the future CPS ones.

- CPS Information Systems, which considers artificial intelligence, data transformation and data analytics to capitalize the huge amount of collected data to reach actionable knowledge.

A brief analysis of reported research and innovation results demonstrated over the last 15-20 years allows to better understand how such actions can be realized by combining CPS, Internet-of-Things and Internet-of-Services technologies. Embedding at large industrial agents and Service-oriented based automation (SOCRADES, 2016; Taisch et al., 2009; Leitão et al., 2016b) is one innovation approach to be highlighted. In fact, agents may act as enablers for CPS-based industrial system architectures and contribute in terms of technology/solution maturity, methodologies and tools, human in the loop, smooth migration and self-* properties, and standardization (Leitão et al., 2016b; Leitão and Karnouskos, 2015a)

Another important dimension for the fully industrial adoption of CPS-based automation systems architectures is the standardization (Kagermann et al., 2013; IEC, 2015), since the standards compliance may affect the development, installation and commissioning of industrial applications. In fact, standardization can support the deployment of CPS, and particularly the smooth migration of these systems, by easily interfacing with existing legacy systems, plugging devices and systems, and adapting their behaviour and relationships on-the-fly. The integration of humans in the loop is seen as a key factor to achieve flexibility (Kagermann et al., 2013), and not more as an obstacle for the complete system automation, as sustained in the past, and particularly during the advent of Computer Integrated Manufacturing (CIM) paradigm.

The Reference Architecture Model Industrie 4.0 (RAMI4.0) standard (DIN, 2016) presents the major architectural specifications for Cyber-Physical components (labelled as I4.0-component) and the set of rules for engineering Industry 4.0 compliant architectures. Aspects related to the CPS-integration within an ISA95compliant architecture, the different phases of the life-cycle of the CPS components and systems of CPS are considered as the base for supporting the engineering of CPS-based industrial systems. In this sense, something that has to be highlighted is the specification of the six digitalization-layers, which cover the full process of building a Cyber-Physical component, starting with the mechatronics (assets) and going through the integration, communication, information, function and business layers. A set of communication and information layers based on the use of Internet technologies, and the exposition of automation function as services in an Internetof-Services fashion, enable the I4.0-components (CPS-component) to engage into business relationships with other components within a system of CPSs.

Additionally, the implementation of the new generation of automation systems will demand new challenges for vocational and academic training and continuing 
professional development, as sustained by the "industry 4.0" high-level working group in its recommended actions (Kagermann et al., 2013; Karnouskos et al., 2014a). In fact, nowadays, engineers need to integrate multidisciplinary and crossdomain knowledge, focusing more on the understanding of system of systems perspective than in a deeply topic domain. In parallel, the penetration of Information and Communication technologies into traditional mechatronics, hydraulics, pneumatic systems, are continuously re-shaping the world, and require an integrative learning process.

The engineering-students are no more dealing only with the physical but predominantly with the cyber part of complex engineering systems, which implies that their acquired knowledge quickly becomes obsolete (some times in less time than the student takes to get the undergraduate degree). Therefore, they need to learn different topics to be able to compete in the future (more systems/system of systems understanding instead of pure (deep) domain knowledge). As example, new engineers have to cope with new paradigms and concepts (e.g., modelling, semantics, (crowd) collaboration, interoperability, self-organization and self-diagnosis) and emergent technologies (e.g., Internet-of-Things, Big data, Machine-to-Machine, advanced data analytics, cloud computing and augmented reality).

Considering all the raised concerns, educating engineers, in the "Industry 4.0" context, means learning how to design, develop, test, deploy, and operate a traditional engineering environment that is being digitalized in both, its structural but also in its behavioural/functional aspects.

The implementation of strategies for the smooth migration from traditional automation systems into the new generation of distributed automation systems are crucial since legacy systems will continue running and will co-exist with the new systems (Leitão et al., 2016b; Karnouskos et al., 2014a). As an example, during the implementation of the GRACE MAS system in the Whirlpool's factory plant producing washing machines (Leitão et al., 2015), the lower control level using PLCs running IEC61313-3 programs was preserved to ensure the real-time control and the MAS solution was placed at the higher control level to introduce intelligence and adaptation to the system performance. However, this is an emergent topic that deserves a significant research in the near future to establish the proper strategies to ensure a smooth migration transforming the existing running systems into Industrie 4.0 compliant systems. These migration strategies should consider the technical perspective, as briefly described in Sect. 8.3.2, but also a deeply study of the impact of economical and social perspectives.

\subsection{Conclusion and Outlook}

There is a need for flexibility, resilience and optimization in industrial settings, that can not be adequately tackled with traditional approaches. Although significant steps have been realized by concepts and utilization of key technologies such as MAS, SOA, Cloud, CPS, significant efforts are still needed to tackle additional challenges related to their engineering and interaction in emerging cooperative 
production systems. The intention in this chapter is not to provide a new modelbased approach but to understand why and how the already existing methods and tools that enable production system flexibility and self-adaptation of CPPS are not adequate or too poorly implemented in industrial practice. At the end, the successful applications of such concepts and technologies will not only be determined by the ability to deal with technology problems, but effectively cover also all other associated aspects that enable continuous business growth and effectiveness.

One of these aspects is about the availability and quality of information. As (sub-) systems are not considered as monolithic building blocks any more, but are seen in their environment of strongly interconnected systems of systems the view on information availability needs to be altered. This altered view needs to reflect not only the system itself, but also its role within its environment, lifecycle, functional hierarchy, etc. This aspect has already been described in Chaps. 5, 6 and 7 of this book.

The increased integration of the cyber and physical aspects of systems, also leads to new challenges for system applications (Lee, 2008; Leitão et al., 2016a; Broy and Schmidt, 2014; Karnouskos et al., 2014a). In the past optimization and improvements have been targeted mainly on isolated parts of the system. Hence, improved production processes and technologies has led to new or improved assets (see top left in Fig. 8.5) or improved control approaches and technologies in the system architecture (see top right in Fig. 8.5). To bring these improvements into

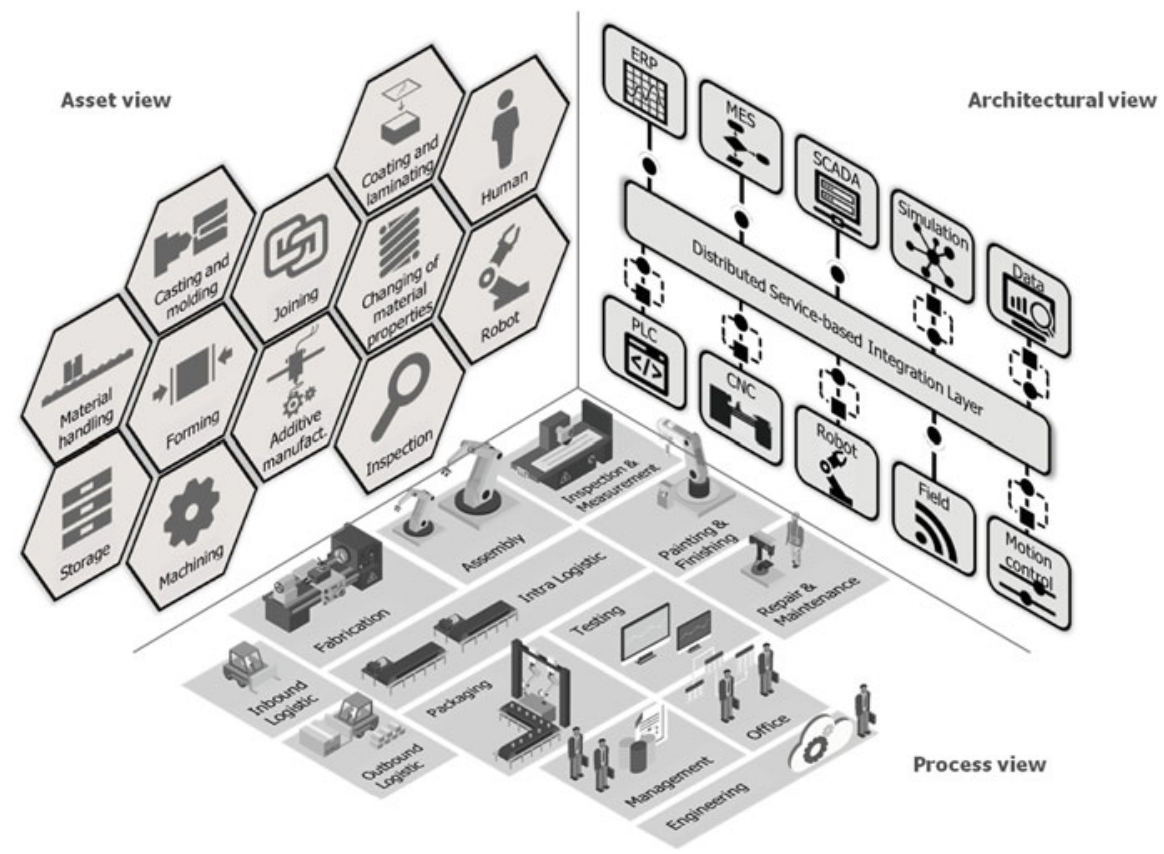

Fig. 8.5 PERFoRM (2016b) project multi-view on production systems 
already existing production systems basically meant to interchange an existing building block (e.g., production asset, IT system) with a new one.

Nowadays these improvements are still possible, but they will not allow to sufficiently address all challenges which are arising from the new complexity of self-* systems and distributed intelligence. In fact the introduction of these concepts requires a change in the heart of each system as they lead to changes in multiple areas and are not isolated only to system building blocks. The integration of assets and IT will allow to improve the whole value adding process (see bottom in Fig. 8.5).

To do this in an efficient and cost-effective way which is suitable for plant operators, new migration methods have to be researched and mitigation strategies need to realized, as discussed in Sects. 8.3 and 8.4. As an example of such an effort, the PERFoRM (2016b) project does not focus on the development of new technologies for tackling flexibility, resilience and optimization needs, but to the re-use of existing developments and their harmonization as also already shown in Sect. 8.2. Additionally a strong focus is set to the development of suitable migration methods and mitigation of existing obstacles in order to create an environment and guidelines for industry to apply decentralized automation system architectures.

This approach, as also proposed within this chapter, allows to re-use already developed technologies and especially to capitalize on the money already spent for this research. Additionally, as a side effect, it stops the ongoing diversification in developed solutions and thus a further diversification of similar technologies to be harmonized or even standardized later on. A downside of this approach is that it can only utilize technologies that already passed at least a conceptional stage at which they are recognized as an already available technology.

Acknowledgements The authors would like to thank the European Commission for the support, and the partners of the EU Horizon 2020 project PERFoRM (2016b) for the fruitful discussions. The PERFoRM project has received funding from the European Union's Horizon 2020 research and innovation programme under grant agreement No 680435.

\section{References}

ACATECH: Cyber-Physical Systems: driving force for innovation in mobility, health, energy and production. Tech. rep., ACATECH - German National Academy of Science and Engineering. https://goo.gl/Q6WFQN (2011)

ANSI/ISA: ANSI/ISA-95.00.01-2010 (IEC 62264-1 Mod) Enterprise-Control System Integration - Part 1: Models and Terminology. http://www.isa.org (2010)

Antzoulatos, N., Castro, E., Scrimieri, D., Ratchev, S.: A multi-agent architecture for plug and produce on an industrial assembly platform. Prod. Eng. Res. Devel. 8(6), 773-781 (2014); doi:10.1007/s11740-014-0571-x

Broy, M., Schmidt, A.: Challenges in engineering Cyber-Physical Systems. Computer 47(2), 70-72 (2014); doi:10.1109/mc.2014.30 
Castellini, P., Cristalli, C., Foehr, M., Leitão, P., Paone, N., Schjolberg, I., Tjonnas, J., Turrin, C., Wagner, T.: Towards the integration of process and quality control using multi-agent technology. In: IECON 2011 - 37th Annual Conference of the IEEE Industrial Electronics Society (2011); doi:10.1109/iecon.2011.6119347

Colombo, A.W., Karnouskos, S.: Towards the factory of the future: a service-oriented crosslayer infrastructure. In: ICT Shaping the World: A Scientific View, pp. 65-81. European Telecommunications Standards Institute/Wiley, New York (2009)

Colombo, A.W., Karnouskos, S., Mendes, J.M.: Factory of the future: a service-oriented system of modular, dynamic reconfigurable and collaborative systems. In: Springer Series in Advanced Manufacturing, pp. 459-481. Springer, London (2010); doi:10.1007/978-1-84996-119-6_15

Colombo, A.W., Karnouskos, S., Bangemann, T.: A system of systems view on collaborative industrial automation. In: 2013 IEEE International Conference on Industrial Technology (ICIT) (2013); doi:10.1109/icit.2013.6505980

Colombo, A.W., Bangemann, T., Karnouskos, S.: IMC-AESOP outcomes: paving the way to collaborative manufacturing systems. In: 2014 12th IEEE International Conference on Industrial Informatics (INDIN) (2014a); doi:10.1109/indin.2014.6945517

Colombo, A.W., Bangemann, T., Karnouskos, S., Delsing, J., Stluka, P., Harrison, R., Jammes, F., Martínez Lastra, J.L. (eds.): Industrial Cloud-based Cyber-Physical Systems: The IMC-AESOP Approach. Springer, New York (2014b)

Delsing, J., Eliasson, J., Kyusakov, R., Colombo, A.W., Jammes, F., Nessaether, J., Karnouskos, S., Diedrich, C.: A migration approach towards a SOA-based next generation process control and monitoring. In: IECON 2011 - 37th Annual Conference of the IEEE Industrial Electronics Society (2011); doi:10.1109/iecon.2011.6120045

Delsing, J., Rosenqvist, F., Carlsson, O., Colombo, A.W., Bangemann, T.: Migration of industrial process control systems into service oriented architecture. In: IECON 2012 38th Annual Conference on IEEE Industrial Electronics Society, Montreal, QC (2012); doi:10.1109/iecon.2012.6389039

DIN: Reference Architecture Model Industrie 4.0 (RAMI4.0). Tech. rep., Deutsches Institut für Normung (DIN). http://www.din.de/en/wdc-beuth:din21:250940128 (2016)

Friedrich, J., Scheifele, S., Verl, A., Lechler, A.: Flexible and modular control and manufacturing system. Procedia CIRP 33, 115-120 (2014); doi:10.1016/j.procir.2015.06.022

Goncalves, G., Reis, J., Pinto, R., Alves, M., Correia, J.: A step forward on intelligent factories: a smart sensor-oriented approach. In: Proceedings of the 2014 IEEE Emerging Technology and Factory Automation (ETFA) (2014); doi:10.1109/etfa.2014.7005227

IEC: White paper factory of the future. Tech. rep., IEC - International Electrotechnical Commission (2015)

Kagermann, H., Wahlster, W., Helbig, J.: Securing the future of German manufacturing industry: recommendations for implementing the strategic initiative industrie 4.0. Tech. rep., ACATECH - German National Academy of Science and Engineering. http://goo.gl/oc3X4n (2013)

Karnouskos, S.: Efficient sensor data inclusion in enterprise services. Datenbank-Spektrum 9(28), 5-10 (2009)

Karnouskos, S.: Realising next-generation web service-driven industrial systems. Int. J. Adv. Manuf. Technol. 60(1-4), 409-419 (2011); doi:10.1007/s00170-011-3612-z

Karnouskos, S., Colombo, A.W.: Architecting the next generation of service-based SCADA/DCS system of systems. In: 37th Annual Conference of the IEEE Industrial Electronics Society (IECON 2011), Melbourne (2011); doi:10.1109/iecon.2011.6119279

Karnouskos, S., Somlev, V.: Performance assessment of integration in the cloud of things via web services. In: 2013 IEEE International Conference on Industrial Technology (ICIT) (2013); doi:10.1109/icit.2013.6505983

Karnouskos, S., Baecker, O., de Souza, L.M.S., Spiess, P.: Integration of SOA-ready networked embedded devices in enterprise systems via a cross-layered web service infrastructure. In: 2007 IEEE Conference on Emerging Technologies and Factory Automation (EFTA 2007), pp. 293 300 (2007); doi:10.1109/efta.2007.4416781 
Karnouskos, S., Guinard, D., Savio, D., Spiess, P., Baecker, O., Trifa, V., de Souza, L.M.S.: Towards the real-time enterprise: service-based integration of heterogeneous SOA-ready industrial devices with enterprise applications. IFAC Proc. Vol. 42(4), 2131-2136 (2009); doi:10.3182/20090603-3-ru-2001.0551

Karnouskos, S., Savio, D., Spiess, P., Guinard, D., Trifa, V., Baecker, O.: Real-World Service Interaction with Enterprise Systems in Dynamic Manufacturing Environments. Springer Series in Advanced Manufacturing, pp. 423-457. Springer, New York (2010); doi:10.1007/978-184996-119-6_14

Karnouskos, S., Colombo, A.W., Bangemann, T.: Trends and challenges for cloud-based industrial cyber-physical systems. In: Industrial Cloud-based Cyber-Physical Systems: The IMC-AESOP Approach, pp. 231-240, Springer, New York (2014a); doi:10.1007/978-3-319-05624-1_11

Karnouskos, S., Colombo, A.W., Bangemann, T., Manninen, K., Camp, R., Tilly, M., Sikora, M., Jammes, F., Delsing, J., Eliasson, J., Nappey, P., Hu, J., Graf, M.: The IMC-AESOP architecture for cloud-based industrial Cyber-Physical Systems. In: Industrial Cloud-Based Cyber-Physical Systems, pp. 49-88. Springer, New York (2014b); doi:10.1007/978-3-319-05624-1_3

Lee, E.A.: Cyber physical systems: design challenges. In: 11th IEEE International Symposium on Object and Component-Oriented Real-Time Distributed Computing (ISORC), pp. 363-369 (2008); doi:10.1109/ISORC.2008.25

Leitão, P., Karnouskos, S.: A survey on factors that impact industrial agent acceptance. In: Industrial Agents: Emerging Applications of Software Agents in Industry, pp. 401-429. Elsevier, Amsterdam (2015a); doi:10.1016/b978-0-12-800341-1.00022-x

Leitão, P., Karnouskos, S. (eds.): Industrial Agents: Emerging Applications of Software Agents in Industry. Elsevier, Amsterdam (2015b)

Leitão, P., Barbosa, J., Vrba, P., Skobelev, P., Tsarev, A., Kazanskaia, D.: Multi-agent system approach for the strategic planning in ramp-up production of small lots. In: 2013 IEEE International Conference on Systems, Man, and Cybernetics (2013); doi:10.1109/smc.2013.807

Leitão, P., Rodrigues, N., Turrin, C., Pagani, A.: Multi-agent system integrating process and quality control in a factory producing laundry washing machines. IEEE Trans. Ind. Inf. 11(4), 879-886 (2015); doi:10.1109/tii.2015.2431232

Leitão, P., Colombo, A.W., Karnouskos, S.: Industrial automation based on cyber-physical systems technologies: Prototype implementations and challenges. Comput. Ind. 81, 11-25 (2016a); doi:10.1016/j.compind.2015.08.004

Leitão, P., Karnouskos, S., Ribeiro, L., Lee, J., Strasser, T., Colombo, A.W.: Smart agents in industrial Cyber-Physical Systems. Proc. IEEE 104(5), 1086-1101 (2016b); doi:10.1109/jproc.2016.2521931

MacKenzie, C.M., Laskey, K., McCabe, F., Brown, P.F., Metz, R.: Reference model for service oriented architecture 1.0. http://docs.oasis-open.org/soa-rm/v1.0/ (2006)

McKinsey: Industry 4.0: How to navigate digitization of the manufacturing sector. Tech. rep., McKinsey Digital. https://www.mckinsey.de/files/mck_industry_40_report.pdf (2015)

Onori, M., Maffei, A., Durand, F.: The IDEAS plug and produce system. In: International Conference on Advanced Manufacturing Engineering and Technologies, NewTech (2013)

PERFoRM: Definition of the system architecture. Tech. rep., Deliverable D2.2, PERFoRM project. http://www.horizon2020-perform.eu/files/documents/D2_2_public.pdf (2016a)

PERFoRM: Production harmonizEd Reconfiguration of Flexible Robots and Machinery (PERFoRM) project, European Commission, horizon 2020 programme. http://www.horizon2020perform.eu (2016b)

Sayed, M.S., Lohse, N., Sondberg-Jeppesen, N., Madsen, A.L.: SelSus: Towards a reference architecture for diagnostics and predictive maintenance using smart manufacturing devices. In: 2015 IEEE 13th International Conference on Industrial Informatics (INDIN), Institute of Electrical and Electronics Engineers (IEEE) (2015); doi:10.1109/indin.2015.7281990

SOCRADES: Service-Oriented Cross-layer infRAstructure for Distributed smart Embedded devices (SOCRADES) project, European Commission, FP6 Programme. http://www.socrades. net (2016) 
Stokic, D., Scholze, S., Barata, J.: Self-learning embedded services for integration of complex, flexible production systems. In: IECON 2011 - 37th Annual Conference of the IEEE Industrial Electronics Society (2011); doi:10.1109/iecon.2011.6119346

Taisch, M., Colombo, A.W., Karnouskos, S., Cannata, A.: SOCRADES roadmap: The future of SOA-based factory automation. Tech. rep., SOCRADES Project. http://www.socrades.net/ Documents/objects/file1274836528.pdf (2009)

Webb, P., Asif, S.: Advanced flexible automation cell. In: 6th Innovation for Sustainable Aviation in a Global Environment (2011)

Wooldridge, M.: An Introduction to Multi-Agent Systems. Wiley, Harlow (2002) 